\title{
中国碳排放效率与产业结构升级的 耦合度及耦合路径
}

\author{
周 迪 ${ }^{1}$, 王雪芹 ${ }^{2}$ \\ (1. 广东外语外贸大学数学与统计学院, 广州 510006; \\ 2. 广东外语外贸大学信息科学与技术学院,广州 510006)
}

\begin{abstract}
摘要: 研究碳排放效率与产业结构升级的耦合关系以及耦合路径, 对新时代中国的绿色发展 以及美丽中国建设具有重要的现实意义。通过包含非期望产出的超效率 SBM 模型测算碳排放 效率, 应用扩展的耦合模型考察了中国各省份碳排放效率与产业结构升级的耦合关系, 并根据 耦合程度和耦合特征对各省份进行多重分类, 在分布动态学框架下设计了中国碳排放效率和 产业结构升级水平的耦合路径。研究发现: (1)中国碳排放效率和产业结构升级水平的时空格 局存在差异, 近一半省份的耦合程度介于 $0.2 \sim 0.4$ 之间, 处于轻度到中度的失调衰退水平, 且近 一半省份的耦合特征属于发展度较低、协调度较高的“低水平协调”类型。(2)从地区间的分布 动态上看, 中国碳排放效率和产业结构升级存在明显的动态不协调, 前者固化程度大于后者, 区域碳排放效率的“低水平陷阱”现象比区域产业结构升级更严重, 故中国应在缓解区域间碳 排放效率固化问题的基础上提升各地区碳排放效率与产业结构升级的耦合度。(3)本文设计的 碳排放效率与产业结构升级的耦合路径, 既能缓解二者在地区之间的动态不协调问题, 又能有 效提升二者在各地区内部的耦合度。
\end{abstract}

关键词: 碳排放效率;产业结构升级;耦合度;固化;耦合路径

中国作为全球碳排放最大的国家, 实现产业结构升级, 既是完成碳减排目标以及实 现中国绿色发展的要求, 又是经济增长速度和质量的决定因素 ${ }^{[1]}$, 能提升碳排放绩效。碳 排放对产业结构产生约束作用，而产业发展对碳排放产生胁迫作用，二者相互影响、相 互作用，构成彼此耦合的交互体。那么当前中国碳排放绩效和产业结构升级具体有着怎 样的耦合关系? 如何更好地提升其耦合水平? 这对于更好地完成中国碳减排目标、促进 中国绿色经济发展具有重要的现实意义。

如何实现碳减排? 目前已经形成共识的是，调整产业结构、改变能源结构和技术进步 是节能减排的三大路径，尤其是产业结构调整，据学者们乐观的估计，中国产业结构调整 对实现碳强度目标的贡献度在 $70 \%$ 以上 ${ }^{[2]}$ 。因此, 关于产业结构调整与升级对碳排放与碳 减排的影响成为国内外学者研究的焦点, 研究内容可分为三类。第一、基于回归模型的 研究。 $\mathrm{He}$ 等 ${ }^{[3]}$ 以及 $\mathrm{Liu}^{[4]}$ 在 $\mathrm{EKC}$ 框架下, 以产业结构为控制变量, 研究其对碳排放的影响; Zhang 等 ${ }^{[5]}$ 采用ARDL 方法分析中国产业结构变化对碳排放强度的影响, 发现第三产业份 额的增加对抑制碳强度起着重要的作用; Wang 等 ${ }^{[6]}$ 采用回归分析方法确定影响中国碳强度 的因素，发现第二产业增加值的比例与碳强度呈正相关。第二、基于能源的视角，采用

收稿日期：2019-04-03; 修订日期：2019-07-11

基金项目：广东省自然科学基金项目（2018A030310044）；全国统计科学研究项目（2017LY55）

作者简介：周迪（1988-), 男, 湖北鄂州人, 博士, 讲师, 研究方向为区域经济、区域资源配置。

E-mail: zhoudi19880101@163.com 
投人产出模型分析产业结构对能源结构、强度、效率等的影响 ${ }^{[7-8]}$ 。第三、通过构建 LMDI (Logarithmic Mean Divisia Index) 模型，从规模、结构以及技术角度进行环境效益分解 ${ }^{[9-10]}$ 。

此外，碳减排政策对产业结构也能产生一定的影响，中国政府制定的碳减排目标对 中国产业结构有一种倒逼机制。除了采取技术手段实现碳减排外，通过经济手段控制二 氧化碳排放也非常重要, 其中最具效率的经济手段包括碳税和碳交易 ${ }^{[11]}$ 。碳市场的建立 和启动, 会对中国部分行业及部分企业的生产产生一定的影响。通过碳排放权的市场交 易, 一方面可实现碳排放控制的目标, 另一方面可促进淘汰落后产能, 实现产业转型升 级, 推动企业的低碳转型, 促进企业的可持续发展, 这对于中国早日实现达峰具有重要 意义, 事实上发达国家工业碳排放达峰正是其产业结构调整和节能减排技术进步作用下 的结果 ${ }^{[12]}$ 。汤维祺等 ${ }^{[13]}$ 梳理了碳减排政策影响地区产业结构的路径, 认为包括生产侧和 需求侧两个方面：从生产侧看，减排政策限制企业的排放，提高能源投人的边际成本， 由于不同行业的碳排放强度不同，生产成本受到的影响也有差异，从而引发资本积累路 径和产业结构的调整; 从需求侧看，政策冲击会通过价格机制向下游产业和消费者传导。

由此可见，产业结构升级能减少碳排放、提升碳排放绩效，同时碳排放政策对产业 结构升级有推动作用，可见碳排放和产业结构之间互相影响，互相作用，构成彼此耦合 的交互系统。但已有相关研究更多关注的是产业结构对碳排放的影响, 就后者对前者影响 的关注相对较少, 而对于二者协调与耦合的研究则更少。耦合模型虽然已较成熟, 但耦合 路径的相关研究却极为滞后。为此, 本文将对中国各省份碳排放绩效与产业结构升级的 耦合关系展开研究, 借助扩展的耦合模型深人地揭示各省份的耦合程度、类型以及特 征, 并首次在分布动态学框架下设计有针对性的耦合路径, 以更好地促进各省份的低碳 经济发展，对新时代中国的绿色发展、美丽中国的建设具有重要的现实意义。

\section{1 研究方法与数据来源}

耦合研究不同于传统的因果关系研究, 其采用系统论的思想全面分析两个系统之间的 相互作用和协同变动关系，传统的耦合模型没有严格区分耦合和协调。逯进等 ${ }^{[14]}$ 首次将其 扩展, 明确地区分了系统的发展和协调两个方面, 认为前者体现了系统从低级到高级的 演进, 后者强调系统内部各要素之间相互配合、和谐发展的程度, 二者共同构成了系统之 间的耦合关系。为此, 本文采用扩展的耦合模型, 首先探索中国各省份 “碳排放效率 一产业结构升级” 两系统的耦合关系与耦合类型, 进而在其基础上引人分布动态学分析框 架，从减缓地区 “低水平陷阱”、实现区域间动态协调的视角设计了耦合路径。

\section{1 耦合模型框架}

(1) 系统发展模型。耦合是协调和发展的统一, 虽然大量学者采用线性函数的形式 来设定系统发展模型, 但这一设定缺乏合理的经济学假定。借鉴逯进等 ${ }^{[14]}$ 的设定方式, 假定发展度函数具有严格的拟凹性以及规模报酬不变性，在这一假设下，发展度函数遵 循柯布一道格拉斯形式, 具体如下:

$$
T=\lambda f(x)^{\alpha} g(y)^{1-\alpha}
$$

式中: $f(x)$ 是归一化后的碳排放效率; $g(y)$ 是归一化后的产业结构升级水平 ${ }^{1}$; $\lambda$ 是外生变 量； $\alpha 、 1-\alpha$ 体现了碳排放效率和产业结构升级在总系统中的重要性程度。

(1) 本文的归一化采用的是 $\min -\max$ 方法, 即 $(x-\min ) /(\max -\min )$ 。 
（2）系统协调模型。借鉴廖重斌 ${ }^{[15]}$ 的研究成果，采用偏离系数刻画系统之间的协调 状况，并在其基础上得到测度系统协调度的模型。借鉴逯进等 ${ }^{[14]}$ 的做法，将偏离系数的 公式转化为:

$$
\begin{gathered}
C_{v}=\sqrt{2(1-C)} \\
C=\left[\frac{4 f(x) g(y)}{(f(x)+g(y))^{2}}\right]^{2}
\end{gathered}
$$

式（3）称为系统的协调度公式。结合图 1 可知, 当某地区两系统的偏离系数 $C_{v}=0$ 时, 协调度 $C=1, f(x)=g(y)$, 此时该地区的坐标点位于从原点出发的 $45^{\circ}$ 线 $C_{0}$ 上。对应 的, $f(x)>g(y)$ 与 $f(x)<g(y)$ 的地区则位于 $C_{0}$ 的下方和上方。对于有相同非零协调度的地区而 言，其坐标点近似地都落在从坐标原点出发、斜率小于或大于 $45^{\circ}$ 的直线上，如图 1 中的 $C_{1}$ 和 $C_{2}$ 直线 ${ }^{2}$; 另外, 坐标点落在 $C_{0}$ 对称的直线上的 地区具有相同的协调度。

（3）耦合模型。根据以上内容可知，协调度 $C$ 衡 量碳排放效率和产业结构升级水平的偏离情况, 但 无法体现二者的发展水平。发展度函数 $T$ 虽然可以衡 量发展水平, 但无法体现二者的协调情况。例如, 一个地区可能存在着协调度较高但发展水平较低的 “低水平协调” 情形（如图 2 中的 $\mathrm{A}$ 点）; 而由于 $C_{1}=$ $C_{2}$, 故 $\mathrm{E}$ 点的协调度和 $\mathrm{D}$ 点一样, 但前者发展度高于 后者； D、B、C 三点虽然有着相同的发展度 $\left(T_{2}\right)$, 但在协调度上存在差异 $(B>D>C)$ 。可见, 各地区 “碳排放效率一产业结构升级” 综合系统的协调和发 展水平存在着各种情形, 仅考察发展度或协调度还 不够, 系统的耦合关系需要能同时考察其发展度和协 调度。为此, 本文的耦合度公式如下:

$$
D=\sqrt{C \times T}
$$

不同的耦合度对应着不同的耦合类型 ${ }^{[15-16]}$, 具体 如表 1 所示。

\section{2 耦合路径思路}

对于耦合度较低的地区, 如何提高其耦合度 呢? 本文首先基于耦合内涵特征进一步对各地区进 行分类，根据协调度和发展度均值将所有地区的耦 合关系划分为四种类型：即协调度较高而发展度较

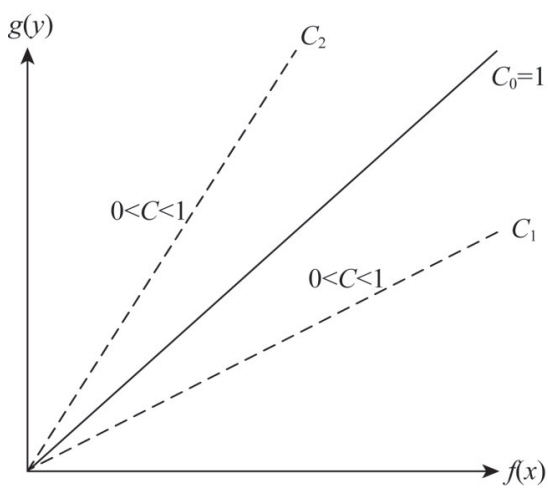

图 1 协调度图形解析

Fig. 1 Graphic analysis of coordination degree

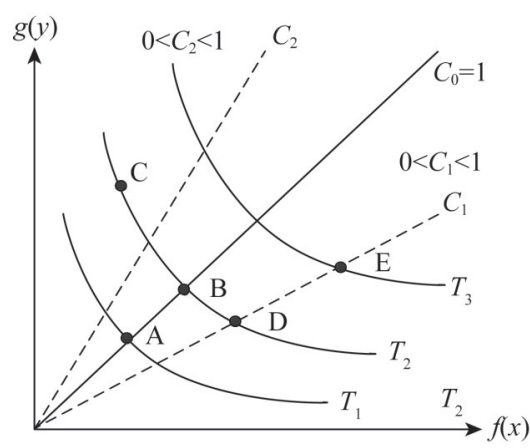

图 2 耦合度图形解析

Fig. 2 Graphic analysis of coupling degree 低（D类型）、协调度和发展度皆较高（A类型）、协调度较低而发展度较高（B类型）、 协调度和发展度皆较低 ( $\mathrm{C}$ 类型)。具体如图 3 所示, 图中的曲线对应考察期内所有地区 的平均发展度，两条从原点出发的射线对应所有地区的平均协调度。

(2) 精确的坐标点构成的其实是从原点出发的一椭圆弧，但由于系统 $f(x)$ 的值经过标准化处理后介于 $0 \sim 1$ 之间, 此时籿圆弧可以近似看为直线，本文通过 Matlab 离散绘图也发现和直线基本一致。逯进等 ${ }^{[14}$ 认为具有相同协调度的 地区的坐标点构成平行于 $45^{\circ}$ 线的直线, 这一结论存在偏误。 
表 1 耦合度对应的耦合类型

Table 1 Coupling types corresponding to coupling values

\begin{tabular}{|c|c|c|c|}
\hline \multicolumn{2}{|c|}{ 负向耦合（失调衰退） } & \multicolumn{2}{|c|}{ 正向耦合（协调发展） } \\
\hline$D$ 值 & 耦合类型 & $D$ 值 & 耦合类型 \\
\hline $0 \sim 0.09$ & 极度失调衰退 & $0.50 \sim 0.59$ & 勉强协调发展 \\
\hline $0.10 \sim 0.19$ & 严重失调衰退 & $0.60 \sim 0.69$ & 初级协调发展 \\
\hline $0.20 \sim 0.29$ & 中度失调衰退 & $0.70 \sim 0.79$ & 中级协调发展 \\
\hline $0.30 \sim 0.39$ & 轻度失调衰退 & $0.80 \sim 0.89$ & 良好协调发展 \\
\hline $0.40 \sim 0.49$ & 濒临失调衰退 & $0.90 \sim 1.00$ & 优质协调发展 \\
\hline
\end{tabular}

在图 3 的分类基础上, 本文在分布动态学框架下 设计了中国 “碳排放效率一产业结构升级” 两系统的 耦合提升路径。分布动态学从长期的角度研究区域事 物分布的动态演进变化, 包括分布的整体动态变化 (核密度曲线刻画) 以及分布的内部动态变化（转移 概率矩阵刻画）两个方面。本文从内部动态变化切 人, 比较地区间碳排放效率以及地区间产业结构升级 的动态变化情况，进而比较二者的区域间 “追赶”与 “固化” 程度。如果前者固化程度严重, 表明中国区 域碳排放 “长期低效率” 相比区域产业结构 “长期低 水平” 现象更严重，区域间的碳排放效率和产业结构 升级动态不协调。因此, 在提升各地区内部碳排放效 率和产业结构升级耦合水平时，政府应该兼顾地区间

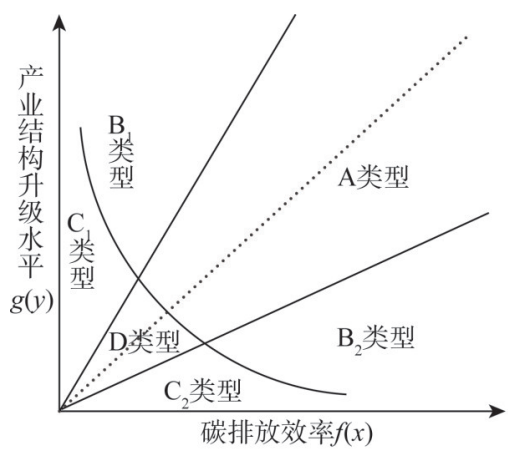

图 3 碳排放效率和产业结构升级堣合 类型

Fig. 3 Coupling types of carbon emission efficiency and industrial structure upgrade 二者的动态协调问题，从固化程度更高的一方人手，对低水平地区加以扶持，优先解决 其 “低水平陷阱”问题。具体是:

路径一：如果区域碳排放效率的固化程度相比产业结构升级更严重，中央政府应该 重点扶持碳排放效率长期处于低水平的地区，例如在 B、C、D类型地区中，应重点扶持 $B_{1} 、 C_{1}$ 类型地区, 特别是地区中碳排放效率较低的省份, 通过提高其碳排放效率来实现 碳排放效率与产业结构升级的协调与发展, 实现 $\mathrm{B}_{1} \rightarrow \mathrm{A} 、 \mathrm{C}_{1} \rightarrow \mathrm{A}$ 的耦合路径, 其意义体现 在两个方面：第一，能解决中国存在的更严重的碳排放效率固化问题，避免碳排放效率 低水平地区陷人 “低水平陷阱”，实现碳排放效率和产业结构升级在我国区域间的动态协 调。第二, 能对 $\mathrm{D}$ 类型地区产生激励效应, 促使其在当前较低的发展水平下, 通过完善 产业结构, 实现产业结构升级, 进人 $\mathrm{B}_{1}$ 或 $\mathrm{C}_{1}$ 类型地区，进而也得到政府的扶持，最后进 人发展度和协调度都较高的 $\mathrm{A}$ 类型地区，从而实现 $\mathrm{D} \rightarrow \mathrm{B}_{1} \rightarrow \mathrm{A}$ 或 $\mathrm{D} \rightarrow \mathrm{C}_{1} \rightarrow \mathrm{A}$ 的耦合路径。

路径二：如果区域产业结构升级的固化程度更严重，此时，中央政府应该重点扶持 产业结构长期处于低水平的地区。具体应该重点扶持 $B_{2} 、 C_{2}$ 类型地区, 走 $B_{2} \rightarrow A$ 或 $C_{2} \rightarrow$ $\mathrm{A}$ 的耦合路径，其意义和路径一类似，不再赘述。

\section{3 碳排放效率、产业结构升级及固化程度的测度方法}

\subsection{1 碳排放效率测度一包含非期望产出的超效率 SBM 模型}

Tone ${ }^{[17]}$ 提出了一种基于松他变量（Slacks-Based Measure，SBM）的 DEA模型，与传 统的 CCR-DEA 及 BBC-DEA 模型相比, SBM 模型直接将松弛变量加人目标函数中, 得到的效率解释是使实际效益最大化，而不是效益比例最大化，属于非径向、非角度的 
效率测度模型。考虑到该模型同样面临着有效决策单元的效率值无法进一步区分的问 题, Tone $\mathrm{e}^{[18]}$ 进一步提出了超效率 SBM 模型。此外, Tone $\mathrm{e}^{[19]}$ 通过将非期望产出纳人 SBM 模 型，可更好地拟合实际生产过程，有效地测算了生产中的环境效率。借鉴已有学者的思 路 ${ }^{200}$, 本文将二氧化碳排放作为非期望产出纳人超效率 SBM 模型中, 以测算碳排放效 率。考虑到包含非期望产出的超效率 SBM 模型较为成熟, 不做具体介绍, 其数学公式可 以参考相关文献 ${ }^{[21]}$ 。

\subsection{2 产业结构升级水平测度}

已有文献主要从产业结构的高级化与合理化来衡量产业结构升级 ${ }^{[22]}$, 由于产业升级 意味着整体产业的变迁和效率的提高, 为了更好地体现本文的研究主旨以及更好契合碳 排放绩效与产业结构升级的耦合内涵，借鉴徐敏等 ${ }^{[23]}$ 的做法，采用产业结构层次指数来 代表产业结构升级水平，其计算公式如下：

$$
\text { upgrade }=\sum_{i=1}^{3} I_{i} \times i=I_{1} \times 1+I_{2} \times 2+I_{3} \times 3
$$

式中: $I_{i}$ 为第 $i$ 产业产值占 GDP 的比例，该指数越大表明地区的产业结构升级水平越高。

\subsection{3 区域固化程度测度一一俱乐部趋同指数}

借鉴周迪等 ${ }^{[24]}$ 提出的俱乐部趋同指数对我国区域碳排放效率和区域产业结构升级的 固化现象和固化程度进行检测和比较分析, 俱乐部趋同指数的构造分两步, 具体如下:

首先, 计算马尔科夫转移概率矩阵: $d$ 年时长的马尔科夫转移概率矩阵的概率值为 $P_{i j}^{t, t+d}=\left\{X_{t+d}=j \mid X_{t}=i\right\}$, 表示在第 $t$ 年、水平处于 $i$ 类型的省份在 $d$ 年后属于 $j$ 类型的概率; 其次，构造可变时长的俱乐部趋同指数： $d$ 年时长的俱乐部趋同指数计算公式如下，该 指数综合了不同类型地区（俱乐部）的规模及其趋同程度。

$$
C C L^{d}=p_{11}^{d} \times r a t_{1}+p_{22}^{d} \times r a t_{2}+\cdots+p_{k k}^{d} \times r a t_{k}
$$

式中： $p_{k k}^{d}$ 为第 $k$ 类俱乐部在 $d$ 年时长下的趋同程度；rat 表示此类俱乐部的规模占比。

\section{4 数据来源}

基于数据的可获得性和实证分析的需要，选取了1997-2016年中国大陆的 29个省 （市、区，简称省份）作为研究对象，其中由于西藏缺乏能源数据而海南缺乏 1952 年的 GDP 数据不予考虑。本文根据投人、期望产出及非期望产出数据计算碳排放效率，并根据 三次产业产值数据计算产业结构升级水平。下面分别对数据处理情况及数据来源进行说明。

在投入数据中，资本投人用资本存量衡量，参考单豪杰 ${ }^{[25}$ 的研究，得到各省份以 1952年为基期的资本存量，缺失数据采用永续盘存法进行估算，估算中采用固定资产投资 价格指数将各省份固定资本形成总额调整为 1952 年为基期的可比价; 劳动力投人用各省份 年末三次产业从业人员总数来衡量; 能源投人采用各省份能源消费总量衡量。产出数据 中，期望产出为各省份 GDP，并将其调整为 1952年为基期的实际 GDP; 非期望产出以各省 份碳排放总量衡量，由于目前国内尚无官方或权威的各省份碳排放数据，故采用杜立民 ${ }^{[26]}$ 的碳排放总量测算方法，根据各省份化石燃料燃烧和水泥的消耗量及对应的碳排放系数 折算得到。具体计算公式为: $C=\sum C_{i} \sum e_{i} \times \varepsilon_{i}$, 其中 $e_{i}$ 为各类资源的消耗量; $\varepsilon_{i}$ 为对应的 $\mathrm{CO}_{2}$ 排放因子, 煤炭、焦炭、汽油、煤油、柴油、燃料油、天然气、水泥的 $\mathrm{CO}_{2}$ 排放系数 分别为 $1.647 \mathrm{t} \mathrm{CO}_{2} / \mathrm{t} 、 2.848 \mathrm{t} \mathrm{CO}_{2} / \mathrm{t} 、 3.045 \mathrm{t} \mathrm{CO}_{2} / \mathrm{t} 、 3.174 \mathrm{t} \mathrm{CO}_{2} / \mathrm{t} 、 3.15 \mathrm{t} \mathrm{CO}_{2} / \mathrm{t} 、 3.064$ $\mathrm{t} \mathrm{CO}_{2} / \mathrm{t} 、 21.670 \mathrm{t} \mathrm{CO}_{2} /$ 亿 $\mathrm{m}^{3} 、 0.527 \mathrm{t} \mathrm{CO}_{2} / \mathrm{t}$ 。能源数据来源于各年《中国能源统计年鉴》, 劳动力数据来源于各省份的统计年鉴, 其他数据全部都来源于国泰安数据库。 


\section{2 结果分析}

\section{1 中国区域碳排放效率和产业结构升级的耦合度分析}

\section{1 .1 碳排放效率和产业升级水平的测算}

采用包含非期望产出的超效率 DEA 模型，本文测算了我国 29 个省份 1997-2016年的 碳排放效率，并计算出各省的产业结构升级水平。借助 ArcGIS 软件，对各省份考察期内 的平均水平进行可视化呈现，并采用自然断点分级法（Natural breaks）划分成四类等 级, 结果如图 4 和图 5 所示。

由图 4 可以看到，我国碳排放效率较低的地区主要为中西部，特别是西北地区，需 要注意的是，云南省的碳排放效率处于第一等级，这可能与该省对高耗能型产业的限制 以及对生态环境的重视有关。东部省份的 碳排放效率相对较高, 所有省份都处于第 二等级以上, 其中上海位于第一等级, 北 京、天津、福建等省份处于第二等级。整 体而言, 胡焕庸线西北侧的效率相对较 低, 而东南侧相对较高。由图 5 可以看 到, 产业结构升级的空间分布与碳排放效 率存在一定的差异, 在自然断点分类下, 第一等级只有两个地区, 分别是北京和上 海; 处于第二等级的地区有天津、广东和 浙江三省份, 可见中国沿海地区省份也普 遍有着较高的产业结构水平。但产业结构 升级水平较低的省份空间分布梯度特征不 明显, 在最低等级中, 既有位于广大中部 地区和西南地区的省份, 也包括位于北方 边境的几个省份。总之, 通过以上分析可 知, 中国碳排放效率与产业结构升级水平 的时间演化及空间分布格局都不太一致， 因此, 有必要对中国各省份碳排放效率和 产业结构升级的协调和耦合关系进行深人 考察, 以更有效地提升各地区的碳排放效 率与产业结构。

2.1 .2 碳排放效率和产业结构升级的耦合度 测算

采用式 (4) 计算各省份逐年的耦合 度 $^{3}$, 需要注意的是, 本文认为产业结构升 级和碳排放效率对当地同等重要, 故令式

(1) 中 $\alpha=0.5, \lambda=1$ 。

结合表 1 中耦合度对应的耦合类型来

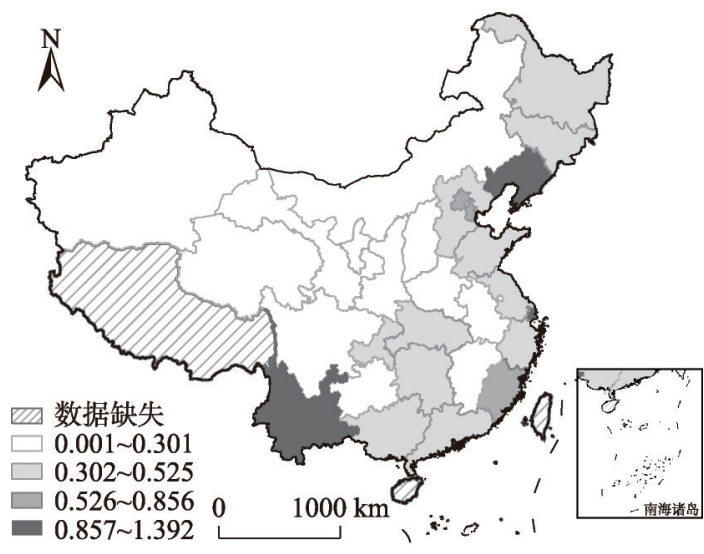

注：本图基于国家测绘地理信息局标准地图服务网站下 载的审图号为 GS(2016)2921 号的标准地图制作，底图无修 改, 下同。

图 4 中国碳排放效率的空间格局

Fig. 4 The spatial pattern of carbon emission efficiency in China

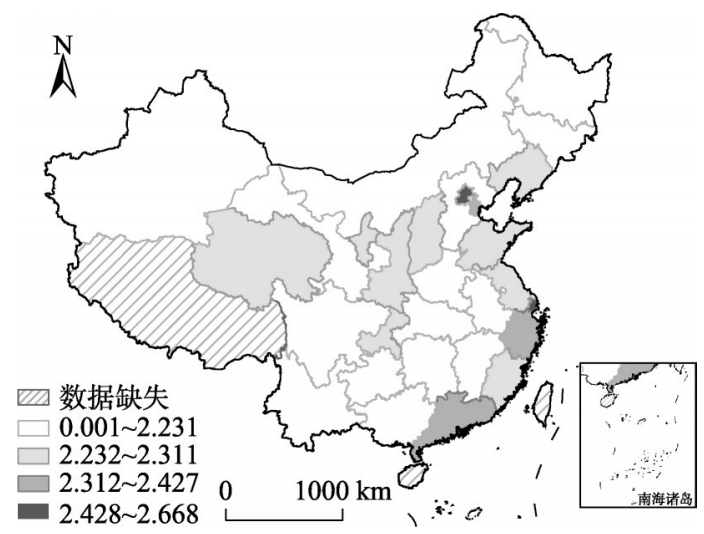

图 5 中国产业结构升级水平的空间格局

Fig. 5 Spatial pattern of industrial structure upgrade level in China

(3) 为节约篇幅, 具体计算结果没有给出, 需要的读者可以向作者索取。 
看, 中国各省份碳排放效率和产业结构升级的耦合度相对较低，处于正向耦合的只有八 个地区，其中上海的耦合度最高，处于优质协调发展状态，其次为天津（中级协调发 展）和北京（初级协调发展），其余五省份则都处于勉强协调发展水平，分别为浙江、福 建、江苏、辽宁和重庆。另外，在失调衰退地区中，处于中度失调衰退 $(0.1 \sim 0.2)$ 的省 份数量最多, 达到八个, 主要为中国西部省份以及少数中部省份; 数量位居第二的是轻 度失调衰退类型（0.3 0.4）地区，包括东北的黑龙江和吉林，西北的甘肃、宁夏和陕西 以及河北省; 处于濒临失调衰退 (0.4 0.5) 的地区有山东、广东、湖北和湖南四省; 耦 合度最低的三个省是：贵州和河南（极度失调衰退类型）以及广西（严重失调衰退类 型）。由此可见，中国整体上各省份碳排放效率和产业结构升级的耦合度较低，且空间分 异明显。另外，从四大地区来看，东部地区的耦合度远大于其他地区，在考察期内都为 0.5 以上的正向耦合，其次为东北地区、中部地区和西部地区。特别地，中西部地区的堣 合度均值分别只有 0.282 和 0.226 , 不及东部地区的一半, 具有较大的提升空间。

从时间上看，除了北京、重庆、四川和安徽等省份的耦合度在上升之外，其他地区 均呈现下降趋势, 其中下降幅度最大的是新疆维吾尔自治区, 其在 1997-2016年间年均下降 $11.409 \%$, 从濒临失调衰退水平（0.405）下降到极度失调衰退水平 $(0.041)$ 。年均下降幅 度较大的省份还包括河南 $(-5.837 \%)$ 、宁夏 $(-5.072 \%)$ 及青海 $(-4.791 \%)$ 。四大地区 的降速与水平呈反比, 西部地区下降速度最快, 其次为中部地区、东北地区和东部地 区，其中东北地区在 2000 年之后开始从正向耦合下降为负向耦合。

综上可见，中国各省份 “碳排放效率一产业结构升级” 系统的耦合程度不仅尚未达 到理想水平, 且普遍有下降的趋势, 这应引起政府的重视。

\section{2 中国区域碳排放效率和产业结构升级的耦合路径}

\subsection{1 中国区域碳排放效率和产业结构的动态协调性分析}

本文从分布的内部动态视角来考察区域间碳排放效率和产业结构升级的协调性，借鉴 周迪等 ${ }^{[24]}$ 在分布动态学框架下构造的俱乐部趋同指数, 测算与比较二者在长时间内高低水 平固化程度的差异。考虑到区域协调政策 的实施需要一定的时间，测算出不同时间 积累下的俱乐部趋同指数, 并且考虑到不 同的离散化方式可能得到不同的分布动态 结果, 本文的俱乐部趋同指数分别在均值 和中位数离散化基础上计算得到, 从而使 结论更稳健。离散化方法借鉴蒲英霞等 ${ }^{[27]}$, 以各年水平的均值或中位数的 $50 \%$ 、 $100 \% 、 150 \%$ 为分界点, 将各地区划分为 四大类型（俱乐部）。其值越大, 表明固化 程度越高, 政府对地区间的调节力度不够, 应该加强调节力度以实现地区间的协调。中 国不同时长下的碳排放效率和产业结构升级 的俱乐部趋同指数测算结果见表 2 。

从表 2 可以看到, 不管采用何种离散 化方式, 中国区域碳排放效率的俱乐部趋

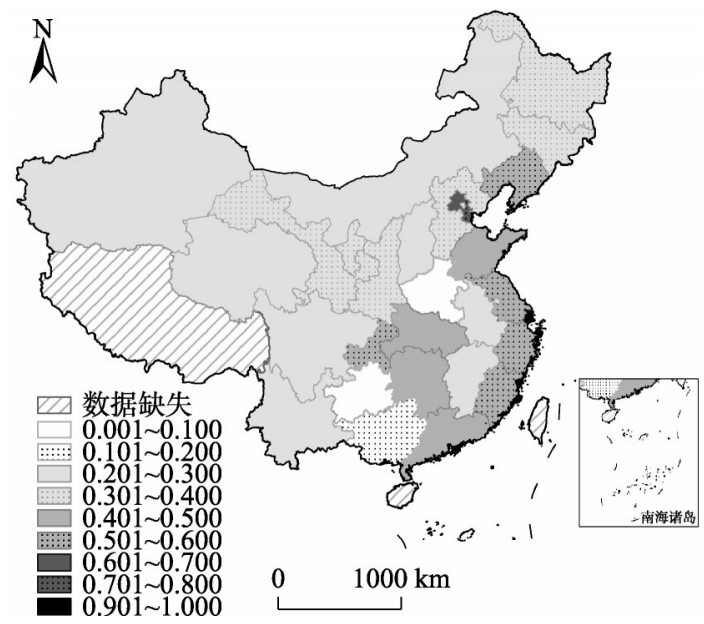

图 6 中国碳排放效率与产业结构升级 耦合度的空间格局

Fig. 6 Spatial pattern of coupling degree between carbon efficiency and industrial structure upgrade in China 
表 2 不同时长下碳排放效率和产业结构升级的俱乐部趋同指数

Table 2 Club convergence index of carbon efficiency and industrial structure upgrade for different durations

\begin{tabular}{|c|c|c|c|c|c|c|}
\hline \multirow{2}{*}{ 时长 } & \multicolumn{3}{|c|}{ 均值划分 } & \multicolumn{3}{|c|}{ 中位数划分 } \\
\hline & 碳排放效率 & 产业结构升级 & 两者之差 & 碳排放效率 & 产业结构升级 & 两者之差 \\
\hline$k=1$ & 0.947 & 0.866 & 0.082 & 0.920 & 0.819 & 0.102 \\
\hline$k=2$ & 0.927 & 0.784 & 0.144 & 0.889 & 0.716 & 0.172 \\
\hline$k=3$ & 0.899 & 0.734 & 0.164 & 0.846 & 0.655 & 0.191 \\
\hline$k=4$ & 0.869 & 0.705 & 0.164 & 0.800 & 0.627 & 0.172 \\
\hline$k=5$ & 0.841 & 0.655 & 0.186 & 0.756 & 0.584 & 0.172 \\
\hline 均值 & 0.897 & 0.749 & 0.148 & 0.842 & 0.680 & 0.162 \\
\hline
\end{tabular}

同指数都较大, 均大于产业结构升级的俱乐部趋同指数, 且这种差异随着时间的积累有 不断增大的趋势。这表明从动态的角度看，区域间碳排放效率和产业结构升级存在明显 的不协调，前者呈现出高低水平固化的特征，而后者的流动性则相对较高，即随着时间 的变化, 一些 “弱势地区” 可能在产业结构升级上表现较好, 呈现出一定的追赶态势, 但这些地区却可能面临着碳排放的 “低效率陷阱” 问题。可见，国家虽然也在强调与重 视能源环境问题以及碳减排问题，但在协调区域间产业升级的效果上要明显好于区域碳 排放效率，这可能与政府对前者采取的 “可行措施” 力度大于后者有一定关系，同时与 中国仍然处于发展中的国情、“发展是硬道理” 等观念有关。今后国家要从加强地区间能 源效率、碳排放效率的协调上努力，既要从观念上重视能源环境问题，也要提出 “切实 可行” 的措施，如对长期处于低水平的地区给予扶持。进一步得到的启示是，在提升中 国碳排放效率和产业结构升级耦合度时，国家应该重点关注因碳排放效率处于低水平而 导致较低耦合度的地区, 要从改善这些地区的碳排放效率人手加以调节, 由此在提升地 区内耦合度的同时，还可以缓解区域间碳排放效率的固化问题。

2.2.2 中国碳排放效率与产业结构升级的耦合路径

（1）中国各地区碳排放效率与产业结构升级的具体塊合类型分类

为了更好地采取措施提升我国碳排放效率和产业结构升级的耦合水平，下面根据中 国各省份碳排放效率和产业结构升级的具体堣合特征对各省份进一步分类。绘制各省份 归一化后的碳排放效率和产业结构升级水平的均值散点图，并根据所有地区协调度水平 以及发展度水平的均值线对各省份进行分类，具体结果如图 7 所示。

图 7 中的曲线为考察期内各省份的平均发展水平线, 关于 $45^{\circ}$ 线对称的两条直线为考 察期内各省份的平均协调水平线，由此中国各地区 “碳排放效率一产业结构升级” 系统 的耦合特征可划分为如下四种类型：

A 类一一发展度和协调度都较高的耦合类型。这类地区包括上海、天津、浙江、江 苏、福建以及重庆等省份。这些地区主要位于中国经济发达的沿海地区，其碳排放效率 和产业结构升级的耦合特征为发展度和协调度均高, 属于中国低碳经济发展的标杆地 区。需要注意的是，上海的耦合度虽然高于天津，但由图 7 可以看到，上海仅在发展度 上高于天津，协调度则低于天津。

$B$ 类一一发展度较高但协调度较低的耦合类型。又可进一步分为 $\mathrm{B}_{1}$ 和 $\mathrm{B}_{2}$ 子类，前者 由于碳排放效率的滞后导致协调度较低, 而后者协调度较低则主要由产业结构升级的滞 后导致。属于前一类型的地区包括云南、辽宁等省份，属于后一类型的地区包括北京、 广东等省份，这类地区应采取更有针对性的措施，补足其低碳经济发展中存在的短板。 
$\mathrm{C}$ 类一一发展度和协调度都较低 的耦合类型。其同样可进一步分为 $\mathrm{C}_{1}$ 和 $\mathrm{C}_{2}$ 子类, 前者较低的协调度主要是 由碳排放效率滞后导致，后者则主要 由产业结构升级的滞后导致。属于 $\mathrm{C}_{1}$ 类的地区包括贵州、青海和山西三 省, 属于 $\mathrm{C}_{2}$ 类的有河南和广西等省 份，这些地区是中国低碳经济发展中 的“弱势地区”，有较大的耦合度提升 潜力和提升空间。

$\mathrm{D}$ 类一一发展度较低但协调度较 高的耦合类型。这些地区包含了中 国 14 个省份，是中国碳排放效率和 产业结构升级耦合关系所属的主要 状态类型，典型地区包括四川、新 疆、安徽、江西、内蒙古等省份， 可见中国各地区的“碳排放效率一

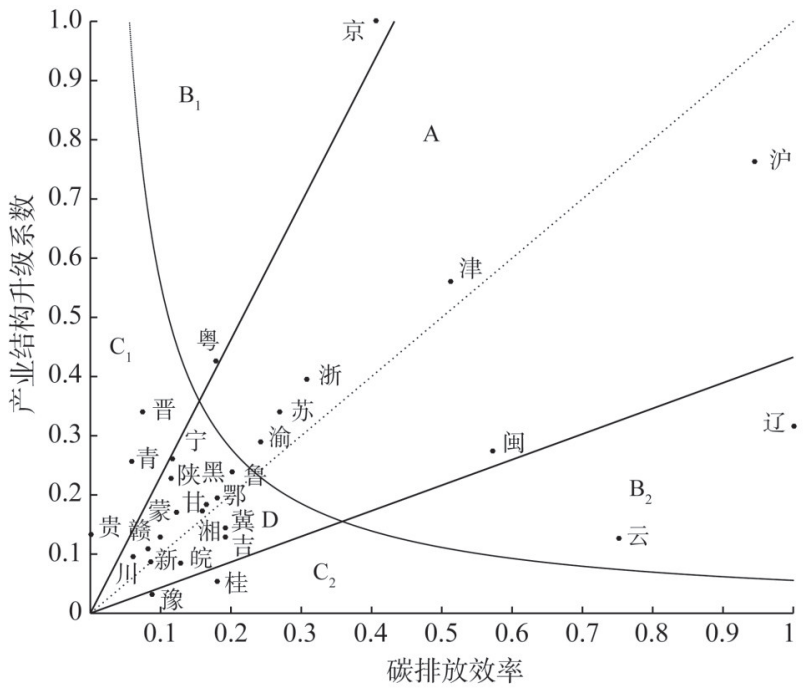

图 7 各地区的碳排放效率和产业结构升级的 具体耦合类型分类

Fig. 7 The classification of coupling types of carbon efficiency and industrial structure upgrade in different regions of China

产业结构升级” 系统存在普遍的 “低水平协调” 现象。导致上述情况的原因可能有两 个：第一、由于各地区的碳排放效率与产业结构升级水平有着相互影响的特点, 故极可 能出现 “双低” 情形，即碳排放效率较低，产业结构升级水平也较低，而 “双低”地区 的协调度能达到较高水平，从而出现 “低水平协调” 情形。这同样表明中国较多地区的 碳排放效率和产业结构升级水平都较低，需要采取措施打破这种低质量协调。第二、这 些 “低水平协调” 地区往往还缺乏打破低水平协调 “僵局” 的动力。因为这些地区在采 取分阶段、分步骤的方式打破 “低水平协调” 时，可能出现更差的结果，即不仅发展度 没有达到应有的高度，反而导致协调度变差。

（2）中国各地区碳排放效率与产业结构升级的堣合路径

考虑到中国碳排放效率的固化程度明显大于产业结构升级，国家应该采取 “路径 一”的耦合提升策略。

具体来说，中央政府应该重点扶持碳排放效率长期处于低水平的地区，应重点支持 $B_{1}$ 和 $C_{1}$ 区域的省份，特别是其中碳排放效率较低的贵州、青海和山西等省份，协助其碳 排放效率的提升，进而提升这些地区的 “碳排放效率一产业结构升级” 系统的协调水 平。另外，由于碳排放效率的提高也能促进系统发展水平的提高，因此这些地区能实现 $\mathrm{C}_{1} \rightarrow \mathrm{A}$ 或 $\mathrm{B}_{1} \rightarrow \mathrm{A}$ 的耦合提升路径。这一策略还具有如下两方面的意义: 第一、能缓解中国 严重的区域碳排放效率固化问题，避免碳排放效率低水平地区陷人 “低水平陷阱”，实现 中国碳排放效率和产业结构的区域间动态协调发展。第二、能对处于 $\mathrm{D}$ 类型地区的省份产 生激励效应，特别是宁夏、陕西、内蒙古、江西、四川等省份。促使其在当前低水平协 调情形下，积极打破协调状态，有步骤、分阶段地提升耦合度。具体地，首先提升产业 结构水平，进人到 $\mathrm{B}_{1}$ 类型区域，或者 $\mathrm{C}_{1}$ 区域 ${ }^{4}$ ，从而也能获得政府的扶持，最后进人发展 
度和协调度都较高的 $\mathrm{A}$ 类型区域，从而走一条 $\mathrm{D} \rightarrow \mathrm{B}_{1} \rightarrow \mathrm{A}$ 或 $\mathrm{D} \rightarrow \mathrm{C}_{1} \rightarrow \mathrm{A}$ 的耦合提升路径。

另外，其他类型地区的省份也可以根据自身的实际情况，采取有针对性的措施提升 其碳排放效率和产业结构升级的耦合水平。例如，对于 $\mathrm{B}_{2}$ 区域的省份而言，其有着较高 的碳排放效率，由于产业结构水平的滞后导致二者的协调度较差。这类地区应该从产业 结构上人手, 通过优化和升级产业结构, 改善和提升经济的发展质量, 提升碳排放效率 和产业结构的协调度，进而进人到具有较高耦合度的 A 区域中。

\section{3 结论}

本文首先采用包含非期望产出的超效率 SBM 模型测算了 1997-2016年中国 29 个省份 的碳排放效率，用产业结构层次指数来测算各省份的产业结构升级水平，进而采用耦合 度模型考察各省份碳排放效率与产业结构升级的耦合度、耦合类型以及具体的耦合特 征，最后在分布动态学（Distribution Dynamics）框架下，从保持区域间碳排放效率与产 业结构升级动态协调的视角提出了二者耦合度的提升路径。研究结论如下:

（1）中国区域碳排放效率整体不高，且呈现下降的趋势，在空间格局上呈现出胡焕 庸线西北低、东南高的特征。产业结构升级水平的空间分异明显, 没有呈现出碳排放效 率的梯度特征，在时间演进上也与碳排放效率不同，整体呈现上升的趋势。可见，中国 碳排放效率与产业结构升级水平的时间演化以及空间分布格局都存在较大差异。

（2）中国碳排放效率和产业结构升级的耦合度较低。除了上海、天津和北京分别处 于优质、中级以及初级协调发展水平状态外, 大部分省份都处于失调衰退状态, 并且以 中度失调衰退和轻度失调衰退为主。另外，在具体的耦合特征上，除了上海、天津、浙 江、江苏、福建以及重庆等省份耦合的发展度和协调度都较高外，接近一半的省份都属 于发展度较低但协调度较高的耦合类型（D)，这些地区应该积极主动采取措施，打破这 种 “低水平协调” 现状，实现耦合度的提升。

（3）中国区域间碳排放效率的固化程度远大于产业结构升级, 二者存在明显的动态 不协调。因此，在提升各省份的耦合度时，中央政府应该对碳排放效率长期处于低水平 的地区进行效率扶持，特别是由于碳排放低效率导致低协调度的地区 $\left(\mathrm{B}_{1} 、 \mathrm{C}_{1}\right)$, 这不仅 能提高该地区的耦合度，还有另外两层意义：第一、能缓解中国区域碳排放效率的固化 问题，特别是 “低效率陷阱” 问题，实现中国碳排放效率与产业结构升级在区域间的动 态协调。第二、能对同样具有碳排放低效率、但产业结构水平也较低的地区（ “低水平 协调” 的 D类型地区）产生激励效应，促使其采取有步骤、分阶段的耦合度提升路径。 另外，各省份应该根据其碳排放效率和产业结构升级耦合特征的具体类型采用有针对性 的改进策略。如碳排放效率较高，但产业结构水平有待提升的地区 $\left(\mathrm{C}_{2}\right)$ 应该重视二者 的协调度水平，可采取单边突破的方式来提高耦合度。

\section{参考文献(References):}

[1] ZHANG J, JIANG H, LIU G, et al. A study on the contribution of industrial restructuring to reduction of carbon emissions in China during the five Five-Year Plan periods. Journal of Cleaner Production, 2018, 176: 629-635.

[2] 王文举, 向其凤. 中国产业结构调整及其节能减排潜力评估. 中国工业经济, 2014, (1): 44-56. [WANG W J, XIANG Q F. Adjustment of industrial structure and the potential assessment of energy saving and carbon reduction. China Idustrial Economics, 2014, (1): 44-56.]

[3] HE J, WANG H. Economic structure, development policy and environmental quality: An empirical analysis of environ- 
mental Kuznets curves with Chinese municipal data. Ecological Economics, 2012, 76(4): 49-55.

[4] LIU L. Environmental poverty, a decomposed environmental Kuznets curve, and alternatives: Sustainability lessons from China. Ecological Economics, 2012, 73: 86-92.

[5] ZHANG Y J, LIU Z, ZHANG H, et al. The impact of economic growth, industrial structure and urbanization on carbon emission intensity in China. Natural Hazards, 2014, 73(2): 579-595.

[6] WANG Z, ZHANG B, LIU T. Empirical analysis on the factors influencing national and regional carbon intensity in China. Renewable \& Sustainable Energy Reviews, 2016, 55: 34-42.

[7] SU B, ANG B W. Multiplicative decomposition of aggregate carbon intensity change using input-output analysis. Applied Energy, 2015, 154: 13-20.

[8] ZHAO R, MIM N, GENG Y, et al. Allocation of carbon emissions among industries/sectors: An emissions intensity reduction constrained approach. Journal of Cleaner Production, 2017, 142: 3083-3094.

[9] ANG B W, SU B. Carbon emission intensity in electricity production: A global analysis. Energy Policy, 2016, 94: 56-63.

[10] 潘文卿, 刘婷, 王丰国. 中国区域产业 $\mathrm{CO}_{2}$ 排放影响因素研究: 不同经济增长阶段的视角. 统计研究, 2017, 34(3): 3044. [PAN W Q, LIU T, WANG F G. A study on factors of regional industrial carbon emission in China: Based on the perspective of different economic growth stages. Statistical Research, 2017, 34(3): 30-44.]

[11] 陈红彦. 基于经济手段的中国低碳制度选择. 广东社会科学, 2013, (2): 252-256. [CHEN H Y. The choice of low carbon system in China based on economic means. Social Sciences in Guangdong, 2013, (2): 252-256.]

[12] 郭朝先. 中国工业碳减排潜力估算. 中国人口·资源与环境, 2014, 24(9): 13-20. [GUO C X. Estimation of industrial carbon emission reduction potential in China. China Population, Resources and Environment, 2014, 24(9): 13-20.]

[13] 汤维祺, 吴力波, 钱浩祺. 从“污染天堂”到绿色增长: 区域间高耗能产业转移的调控机制研究. 经济研究, 2016, 51 (6): 58-70. [TANG W Q, WU L B, QIAN H Q. From Pollution-heaven to green-growth: Impact of carbon-market relocation of energy-intensive-sectors. Economic Research Journal, 2016, 51(6): 58-70.]

[14] 逯进, 周惠民. 中国省域人力资本与经济增长耦合关系的实证分析. 数量经济技术经济研究, 2013, 30(9): 3-19. [LU J, ZHOU H M. Empirical analysis of coupling relationship between human capital and economic growth in Chinese provinces. The Journal of Quantitative \& Technical Economics, 2013, 30(9): 3-19.]

[15] 廖重斌. 环境与经济协调发展的定量评判及其分类体系: 以珠江三角洲城市群为例. 热带地理, 1999, 10(2): 76-82. [LIAO C B. Quantitative gudgement and classificationl system for coordinated development of environment and economy: A case study of the city group in the Pearl River Delta. Tropical Geography, 1999, 10(2): 76-82.]

[16] 吴文恒, 牛叔文, 郭晓东, 等. 中国人口与资源环境耦合的演进分析. 自然资源学报, 2006, 21(6): 853-861. [WU W H, NIU S W, GUO X D, et al. Evolutional analysis of coupling between poputation and resource-environment in China. Journal of Natural Resources, 2006, 21(6): 853 -861.]

[17] TONE K. A slacks-based measure of efficiency in data envelopment analysis. European Journal of Operational Research, 2001, 130(3): 498-509.

[18] TONE K. A slacks-based measure of super-efficiency in data envelopment analysis. European Journal of Operational Research, 2002, 143(1): 32-41.

[19] TONE K. Dealing with undesirable outputs in DEA: A Slacks Based Measure (SBM) approach. GRIPS Research Report Seires, 2003-0005.

[20] 周五七, 聂鸣. 中国工业碳排放效率的区域差异研究: 基于非参数前沿的实证分析. 数量经济技术经济研究, 2012, 29(9): 58-70. [ZHOU W Q, NIE M. Regional differences in the efficiency of industrial carbon emissions in China. The Journal of Quantitative \& Technical Economics, 2012, 29(9): 58-70.]

[21] 宫大鹏, 赵涛, 慈兆程, 等. 基于超效率 SBM 的中国省际工业化石能源效率评价及影响因素分析. 环境科学学报, 2015, 35(2): 585-595. [GONG D P, ZHAO T, CI Z C, et al. Evaluation of regional industrial fossil energy efficiency in China based on super SBM and factors analysis. Acta Scientiae Circumstantiae, 2015, 35(2): 585-595.]

[22] 干春晖, 郑若谷, 余典范. 中国产业结构变迁对经济增长和波动的影响. 经济研究, 2011, 46(5): 4-161. [GAN C H, ZHEGN R G, YU D F. Cyclical fluctuations and nonlinear dynamics of inflation rate. Economic Research Journal, 2011, 46(5): 4-161.]

[23] 徐敏, 姜勇. 中国产业结构升级能缩小城乡消费差距吗?. 数量经济技术经济研究, 2015, 32(3): 3-21. [XU M, JIANG Y. Can the China's industrial structure upgrading narrow the gap between urban and rural consumption?. The Journal of Quantitative \& Technical Economics, 2015, 32(3): 3-21.]

[24] 周迪, 周丰年. 中国水资源利用效率俱乐部趋同的检验、测度及解释: 2003-2015 年. 自然资源学报, 2018, 33(7): 1103-1115. [ZHOU D, ZHOU F N. Inspection, measurement and interpretation of club convergence of water resources utilization efficiency in China: 2003-2015. Journal of Natural Resources, 2018, 33(7): 1103-1115.]

[25] 单豪杰. 中国资本存量 K 的再估算: 1952-2006年. 数量经济技术经济研究, 2008, 25(10): 17-31. [SHAN H J. Reesti- 
mating the capital stock of China: 1952-2006. The Journal of Quantitative \& Technical Economics, 2008, 25(10): 17-31.]

[26] 杜立民. 我国二氧化碳排放的影响因素: 基于省级面板数据的研究. 南方经济, 2010, (11): 20-33. [DU L M. Impact factors of China's carbon dioxide emissions: Provincial panel data analysis. South China Journal of Economics, 2010, (11): 20-33.]

[27] 蒲英霞, 马荣华, 葛芗, 等. 基于空间马尔可夫链的江苏区域趋同时空演变. 地理学报, 2005, 60(5): 817-826. [PU Y X, MA R H, GE Y, et al. Spatial-temporal dynamics of jiangsu regional convergence with spatial markov chains approach. Acta Geographica Sinica, 2005, 60(5): 817-826.]

\title{
Research on coupling degree and coupling path between China's carbon emission efficiency and industrial structure upgrade
}

\author{
ZHOU Di ${ }^{1}$, WANG Xue-qin ${ }^{2}$ \\ (1. School of Mathematics and Statistics, Guangdong University of Foreign Studies, Guangzhou 510006, China; \\ 2. School of Information Science and Technology, Guangdong University of Foreign \\ Studies, Guangzhou 510006, China)
}

\begin{abstract}
The study of the coupling relationship and coupling path of carbon emission efficiency and industrial structure upgrade is of great practical significance to the green development of China in the new era and for the construction of a beautiful China. Firstly, the carbon emission efficiency is calculated by the super-efficient SBM model containing undesired output. Then, the coupling degree between the efficiency of regional carbon emission and the industrial structure upgrade is calculated and analyzed by the extended coupling model, and the provinces are divided into multiple layers according to their coupling degrees and coupling characteristics. Finally, the coupling path of the two factors is designed under the framework of dynamic distribution. The research found that: (1) There lies an obvious difference between the spatial and temporal pattern of carbon emission efficiency and high-grade industrial structure level. In terms of their coupling degrees, nearly half of the provinces are between 0.2 and 0.4 , which can be considered as moderately and mildly imbalanced recession. Besides, according to the coupling characteristics, nearly half of the provinces belong to the coupling type of "low level coordination" with low development degree and high coordination degree. (2) There is an obvious inconsistency between carbon emission efficiency and the industrial structure upgrade in China from the aspect of reginal dynamic distribution. The former has a severer degree of cure than the latter. Therefore, China should improve the degree of coupling between regional carbon emission efficiency and industrial structure on the basis of mitigating the solidification of interregional carbon emission efficiency. (3) The paper designs the coupling path of carbon emission efficiency and the industrial structure upgrade in China on the basis of the coupling classification diagram, which can not only alleviate the dynamic incoordination of the two factors, but also effectively enhance their coupling degree among regions of China.
\end{abstract}

Keywords: carbon emission efficiency; industrial structure upgrade; coupling degree; cure; coupling path 